\title{
ASSOCIO-SYMMETRIC ALGEBRAS
}

\author{
BY \\ RAYMOND COUGHLIN AND MICHAEL RICH( $\left.{ }^{1}\right)$
}

\begin{abstract}
Let $A$ be an algebra over a field $F$ satisfying $(x, x, x)=0$ with a function $g: A \times A \times A \rightarrow F$ such that $(x y) z=g(x, y, z) x(y z)$ for all $x, y, z$ in $A$. If $g\left(x_{1}, x_{2}, x_{3}\right)$ $=g\left(x_{1 \pi}, x_{2 \pi}, x_{3 \pi}\right)$ for all $\pi$ in $S_{3}$ and all $x_{1}, x_{2}, x_{3}$ in $A$ then $A$ is called an associosymmetric algebra. It is shown that a simple associo-symmetric algebra of degree $>2$ or degree $=1$ over a field of characteristic $\neq 2$ is associative. In addition a finitedimensional semisimple algebra in this class has an identity and is a direct sum of simple algebras.
\end{abstract}

Throughout we shall let $A$ denote an associo-symmetric algebra over a field $F$ of characteristic $\neq 2$. In $\S 1$ we show that $A$ is power-associative and has a vector space decomposition $A=A_{11}+A_{10}+A_{01}+A_{00}$ relative to any idempotent $e$. In $\S 2$ the multiplicative properties of the submodules are studied and as a consequence of these one obtains Theorem 3.2 that if $A$ is simple and $e \neq 1$ is an idempotent then $A_{11}(e)$ and $A_{00}(e)$ are associative subalgebras. The decomposition of $A$ relative to several orthogonal idempotents, derived in $\$ 4$, is used to obtain Theorem 4.2 that if $A$ is simple and has degree $>2$ then $A$ is associative. The main result of $\S 5$ is that if $A$ is finite dimensional and semisimple then $A$ has an identity and is a direct sum of simple algebras. Finally in $\S 6$ an argument is adopted from alternative rings to show that if $A$ is simple and of degree one then it is a field.

\section{Preliminaries.}

THEOREM 1.1. If $A$ is an associo-symmetric algebra then $A$ is power-associative.

Proof. We show that $x^{a} x^{b}=x^{a+b}$ for any $x$ in $A$ by induction on $k=a+b$. The result holds if $k=3$ by third power-associativity. Assume that the result holds for all $k<n$ and let $0<s \leqq n-1$. Then $x^{n-1} x=\left(x^{n-s-1} x^{s}\right) x=g\left(x^{n-s-1}, x^{s}, x\right) x^{n-s-1}\left(x^{s+1}\right)$. On the other hand $x^{n-s} x^{s}=\left(x^{n-s-1} x\right) x^{s}=g\left(x^{n-s-1}, x, x^{s}\right) x^{n-s-1} x^{s+1}$. By associosymmetry, however, $g\left(x^{n-s-1}, x^{s}, x\right)=g\left(x^{n-s-1}, x, x^{s}\right)$. Therefore $x^{n-1} x=x^{n-s} x^{s}$ and if we let $a=n-s, b=s$ then $x^{a} x^{b}=x^{n}=x^{a+b}$. Thus, $A$ is power-associative by finite induction.

It seems worthwhile to remark here that the assumption $(x y) z=g(x, y, z) x(y z)$ with $g: A \times A \times A \rightarrow F$ and $g\left(x_{1}, x_{2}, x_{3}\right)=g\left(x_{1 \pi}, x_{2 \pi}, x_{3 \pi}\right)$ is not in itself sufficient to

Received by the editors August 11, 1970.

AMS 1969 subject classifications. Primary 1720.

Key words and phrases. Associo-symmetric, power-associative, orthogonal idempotents, semisimple, degree, principal idempotent.

${ }^{(1)}$ The second author was partially supported by a Temple University research grant. Copyright (C) 1972, American Mathematical Society 
guarantee power-associativity even in finite dimension, as the following example indicates. Let $A$ have basis $a, b, c, d, e, f$ over a field of characteristic $\neq 2$ with multiplication given by $a b=c, c d=e, b d=2 f$, $a f=e$, and all other products zero. Then $g(x, y, z)=\frac{1}{2}$ for all $x, y, z$ in $A$. However

$$
(a+b+d)^{2}(a+b+d)=(c+2 f)(a+b+d)=e
$$

and

$$
(a+b+d)(a+b+d)^{2}=(a+b+d)(c+2 f)=2 e .
$$

Therefore $A$ is not power-associative.

Lemma 1.1. Let $e$ be an idempotent of an associo-symmetric algebra $A$ over a field of characteristic $\neq 2$. Then $(a, e, e)=(e, a, e)=(e, e, a)=0$ for all $a$ in $A$. $($ Here $(a, b, c)=(a b) c-a(b c)$.)

Proof. Since $A$ is power-associative, $A$ has a vector space decomposition $A=A_{1}+A_{1 / 2}+A_{0}$ relative to $e$ where $A_{i}=\left\{a_{i} \mid e a_{i}+a_{i} e=2 i a_{i}\right\}$ for $i=1,0, \frac{1}{2}[1]$. Since $A$ satisfies $(x, x, x)=0$ it can be shown (see [6, p. 137]) that

$$
A_{i}=\left\{a_{i} \mid e a_{i}=a_{i} e=i a_{i}\right\} \text { for } i=1,0 .
$$

Now let $a \neq 0$ be in $A$. From $(x, x, x)=0$ we have $(e, e, a)+(e, a, e)+(a, e, e)=0$. If we let $\alpha=g(e, e, a)=g(a, e, e)=g(e, a, e)$ then by the associo-symmetric identity, $(\alpha-1) e(e a)+(\alpha-1) e(a e)+(\alpha-1) a e=0$. If $\alpha=1$ the lemma follows. Otherwise $e(e a)+e(a e)+a e=0$. Let $a=a_{1}+a_{1 / 2}+a_{0}$. Then $a e=a_{1}+a_{1 / 2} e, e(a e)=a_{1}+e\left(a_{1 / 2} e\right)$, and $e(e a)=a_{1}+e\left(e a_{1 / 2}\right)$. Thus we have $3 a_{1}+a_{1 / 2} e+e\left(a_{1 / 2} e+e a_{1 / 2}\right)=0$. But $a_{1 / 2} e$ $+e a_{1 / 2}=a_{1 / 2}$. Therefore we get $3 a_{1}+a_{1 / 2}=0, a_{1 / 2}=0$ and $a=a_{1}+a_{0}$. (In fact if characteristic $F \neq 3$ then $a=a_{0}$.) Thus $(e, a, e)=(a, e, e)=(e, e, a)=0$.

It is a well-known fact that in any algebra $A$, the results of Lemma 1.1 imply that $A$ has a Peirce decomposition $A=A_{11}+A_{10}+A_{01}+A_{00}$. Therefore we have

THEOREM 1.2. If $A$ is an associo-symmetric algebra over a field of characteristic $\neq 2$ and if $e$ is an idempotent of $A$ then $A=A_{11}(e)+A_{10}(e)+A_{01}(e)+A_{00}(e)$ where $A_{i j}(e)=\left\{x_{i j} \mid e x_{i j}=i x_{i j}\right.$ and $\left.x_{i j} e=j x_{i j}\right\}$.

It is clear that if $a=a_{11}+a_{10}+a_{01}+a_{00}$ then $a_{11}=e a e, a_{10}=e a-e a e, a_{01}=a e$ $-e a e$, and $a_{00}=a-a e-e a+e a e$.

\section{Multiplication of the modules.}

LEMMA 2.1. $\left(A_{11}+A_{01}\right)\left(A_{00}+A_{01}\right)=0$.

Proof. Let $x \in A_{11}+A_{01}, y \in A_{00}+A_{01}$. Then $x y=(x e) y=g(x, e, y) x(e y)=0$.

Lemma 2.2. $A_{11} A_{11} \subseteq A_{11}, A_{11} A_{10} \subseteq A_{10}$.

Proof. Let $x, y \in A_{11}$ and $g(e, x, y)=\alpha$. Then $x y=(e x) y=\alpha e(x y)$. If $\alpha=0$ then $x y=0 \in A_{11}$. Suppose $\alpha \neq 0$ and $x y=a_{11}+a_{10}+a_{01}+a_{00}$. Then $x y=\alpha\left(a_{11}+a_{10}\right)$ or $a_{11}+a_{10}+a_{01}+a_{00}=\alpha\left(a_{11}+a_{10}\right)$. The vector space direct sum then forces $\alpha=1$ 
and $a_{01}=a_{00}=0$. Therefore $x y \in A_{11}+A_{10}$. On the other hand $g(x, y, e)=\alpha=1$, $(x y) e=x y$. Therefore $x y \in A_{11}+A_{01}$. Thus, $x y \in\left(A_{11}+A_{10}\right) \cap\left(A_{11}+A_{01}\right)=A_{11}$.

Now, let $x \in A_{11}, y \in A_{10}$ and $\alpha=g(x, e, y)$. Then $x y=(x e) y=\alpha x(e y)=\alpha x y$. If $\alpha=0$ then $x y=0 \in A_{10}$. Otherwise $\alpha=1=g(e, x, y)$. Thus $x y=(e x) y=e(x y)$ and $x y \in A_{10}+A_{11}$. However, $(x y) e=x(y e)=0$. Therefore $x y \in A_{10}+A_{00}$. Thus, $x y \in A_{10}$.

Lemma 2.3. $A_{01} A_{11} \subseteq A_{01}, A_{01} A_{10} \subseteq A_{00}$.

Proof. Let $x \in A_{01}, \quad y \in A_{11}, \quad \alpha=g(x, e, y)$. Then $x y=(x e) y=\alpha x(e y)=\alpha x y$. Therefore $\alpha=0$ or 1. If $\alpha=0$ then $x y=0 \in A_{01}$. Otherwise $\alpha=1=g(e, x, y)$ $=g(x, y, e)$. Therefore $0=(e x) y=e(x y)$ and $(x y) e=x(y e)=x y$. Thus, $x y \in\left(A_{01}+A_{00}\right)$ $\cap\left(A_{01}+A_{11}\right)=A_{01}$.

Next let $x \in A_{01}, y \in A_{10}$. Clearly $x y \in A_{10}+A_{00}$ since $(x y) e=g(x, y, e) x(y e)=0$. Let $\alpha=g(x, e, y)=g(e, x, y)$. If $\alpha=0$ then $(x e) y=0$ and $x y=0 \in A_{00}$. Otherwise $g(e, x, y) \neq 0$. Then $0=(e x) y=g(e, x, y) e(x y)$ and $e(x y)=0$. Thus $x y \in A_{01}+A_{00}$. But $x y \in A_{10}+A_{00}$. Therefore $x y \in A_{00}$.

Lemma 2.4. $A_{10}\left(A_{10}+A_{11}\right)=0, A_{10} A_{00} \subseteq A_{10}, A_{10} A_{01} \subseteq A_{11}$.

Proof. Let $x \in A_{10}, y \in A_{1 i}$ for $i=0,1$ and $\alpha=g(x, e, y)=g(e, x, y)$. Then $0=(x e) y=\alpha x(e y)=\alpha x y$. If $\alpha \neq 0$ then $x y=0$. Otherwise $\alpha=g(e, x, y)=0$. Then $x y=(e x) y=0 e(x y)=0$. Therefore $x y=0$. Next let $x \in A_{10}, y \in A_{00}$. Then $x y=(e x) y=$ $\alpha e(x y)$. As in the proof of Lemma 2.2 this forces $\alpha=0$ or 1. If $\alpha=0$ we are done. If $\alpha=1$ then $x y \in A_{10}+A_{11}$. But $(x y) e=0$. Therefore $x y \in A_{10}+A_{00}$. Hence, $x y \in A_{10}$.

Finally, let $x \in A_{10}, y \in A_{01}$. Then $x y=(e x) y=\alpha e(x y)$. Again, $\alpha=0$ or 1. If $\alpha=0$ then $x y=0 \in A_{11}$. Otherwise $\alpha=1,(x y) e=x(y e)=x y$ and $x y=(e x) y=e(x y)$. Thus $x y \in A_{11}$.

Lemma 2.5. $A_{00}\left(A_{10}+A_{11}\right)=0, A_{00} A_{01} \subseteq A_{01}, A_{00} A_{00} \subseteq A_{00}+A_{10}$.

Proof. Let $x \in A_{00}, y \in A_{1 i}$ for $i=0,1$. If $\alpha=1$ then $0=(x e) y=x(e y)=x y$. Otherwise $\alpha \neq 1$. Linearization of third power-associativity gives $(x, e, y)+(x, y, e)$ $+(y, x, e)+(y, e, x)+(e, x, y)+(e, y, x)=0$ or

$$
(\alpha-1)[x(e y)+x(y e)+y(x e)+y(e x)+e(x y)+e(y x)]=0 .
$$

Since $\alpha \neq 1$ and by the definition of the modules, we have

$$
x y+i(x y)+e(x y)+e(y x)=0 .
$$

If $i=1$ then $y x \in A_{11} A_{00}=0$ by Lemma 2.1. Therefore $-\frac{1}{2} e(x y)=x y$, which forces $x y=0$. If $i=0$ then reconsider $\alpha$. If $\alpha=g(x, e, y) \neq 0$ then $0=(x e) y=\alpha x(e y)$ $=\alpha x y$. Therefore $x y=0$. Otherwise $\alpha=0=g(e, y, x)$ and $y x=(e y) x=0$. Therefore (1) reduces to $x y+e(x y)=0$. This again forces $x y=0$ to show that $A_{00}\left(A_{10}+A_{11}\right)$ $=0$. 
Now let $x \in A_{00}, y \in A_{01}$. If $\alpha=1$ then $(x y) e=x(y e)=x y$ and $e(x y)=(e x) y=0$. Therefore $x y \in A_{01}$. If $\alpha \neq 1$ then by a linearization of third power-associativity as in Lemma 2.5 we have $x(e y)+x(y e)+y(x e)+y(e x)+e(x y)+e(y x)=0$ which reduces to $x y+e(x y)+e(y x)=0$ since $x \in A_{00}, y \in A_{01}$. Now $y x \in A_{01} A_{00}=0$ by Lemma 2.1. Therefore $x y+e(x y)=0$ which forces $x y=0 \in A_{01}$. Therefore $A_{00} A_{01}$ $\subseteq A_{01}$. Finally, the last statement of Lemma 2.5 is immediate.

The results of Lemmas $2.1-2.5$ give the following.

THEOREM 2.1. If $e$ is an idempotent of an associo-symmetric algebra $A$ over $a$ field of characteristic $\neq 2$ then the modules $A_{i j}(e)$ have the multiplicative relations

(2) $A_{11} A_{11} \subseteq A_{11}$,

(3) $A_{00} A_{00} \subseteq A_{00}+A_{10}$,

(4) $A_{i j} A_{k l}=0$ if $j \neq k$,

(5) $A_{i j} A_{j l} \subseteq A_{i l}$ unless $i=j=l=0$.

It should be noted that if $A$ has an identity 1 and $e \neq 1$ then (3) can be strengthened to $A_{00}(e)^{2} \subseteq A_{00}(e)$. For $A_{00}(e)^{2}=A_{11}(1-e)^{2} \subseteq A_{11}(1-e)=A_{00}(e)$.

3. Simple algebras. In an associative algebra the set $B=A_{10} A_{01}+A_{10}+A_{01}$ $+A_{01} A_{10}$ is an ideal. We prove the same result for associo-symmetric algebras and use it to characterize the simple algebras. According to convention "simple" means "simple but not nil".

LEMMA 3.1. $A_{11}\left(A_{10} A_{01}\right) \subseteq A_{10} A_{01}$.

Proof. Let $x \in A_{11}, y \in A_{10}, z \in A_{01}$. If $\alpha=g(x, y, z)=g(x \pi, y \pi, z \pi)=1$ then $x(y z)=(x y) z \in A_{10} A_{01}$ by (5). Otherwise the linearization of $(a, a, a)=0$ gives

$$
x(y z)+x(z y)+y(x z)+y(z x)+z(x y)+z(y x)=0 .
$$

Now $x(z y) \in A_{11} A_{00}=0, x z \in A_{11} A_{01}=0$, and $y x \in A_{10} A_{11}=0$ by (4). Therefore (6) reduces to $x(y z)+y(z x)+z(x y)=0$. But $x(y z) \in A_{11}, y(z x) \in A_{11}$, and $z(x y) \in A_{00}$. Therefore $z(x y)=0$ and (6) reduces to $x(y z)=-y(z x)$. But $y(z x) \in A_{10}\left(A_{01} A_{11}\right)$ $\subseteq A_{10} A_{01}$ by (5). Therefore $x(y z) \in A_{10} A_{01}$.

Lemma 3.2. $A_{00}\left(A_{01} A_{10}\right) \subseteq A_{01} A_{10}$.

Proof. Let $x \in A_{00}, y \in A_{01}, z \in A_{10}$. If $g(x, y, z)=1$ then $x(y z)=(x y) z \in A_{01} A_{10}$. Otherwise we have (6). But $x(z y) \in A_{00} A_{11}=0, x z \in A_{00} A_{10}=0$ and so $y(x z)=0$, and $z(y x) \in A_{10}\left(A_{01} A_{00}\right)=0$. Therefore (6) reduces to $x(y z)+z(x y)+y(z x)=0$. But $x(y z) \in A_{00}\left(A_{01} A_{10}\right) \subseteq A_{00}^{2} \subseteq A_{00}+A_{10}, y(z x) \in A_{01} A_{10} \subseteq A_{00}$, and $z(x y) \in A_{10} A_{01}$ $\subseteq A_{11}$. Therefore $z(x y)=0$ and $x(y z)=-y(z x) \in A_{01} A_{10}$ to prove the lemma.

THEOREM 3.1. In any associo-symmetric algebra $A$ with idempotent $e, B=A_{10} A_{01}$ $+A_{10}+A_{01}+A_{01} A_{10}$ is an ideal of $A$.

Proof. Since $A=\sum_{i, j=0,1} A_{i j}$ it is sufficient to show that $A_{i j} B+B A_{i j} \subseteq B$ for $i, j=0,1$. Now the multiplicative properties in Theorem 2.1 and associo-symmetry 
immediately imply that $B A_{i j} \subseteq B$ for $i, j=0,1$. Similarly, Theorem 2.1 implies that $A_{10} B+A_{01} B \subseteq B$. Consider $A_{11} B=A_{11}\left(A_{10} A_{01}+A_{10}+A_{01}+A_{01} A_{10}\right)$. Now $A_{11} A_{01}$ $=A_{11}\left(A_{01} A_{10}\right)=0, A_{11} A_{10} \subseteq A_{10} \subseteq B$ by Theorem 2.1 and $A_{11}\left(A_{10} A_{01}\right) \subseteq A_{10} A_{01} \subseteq B$ by Lem ina 3.1. We similarly use Lemma 3.2 to show that $A_{00} B \subseteq B$. Thus $B$ is an ideal of $A$.

Corollary 1. If $e \neq 1$ is an idempotent of a simple associo-symmetric algebra $A$ then $A_{11}(e)=A_{10}(e) A_{01}(e)$ and $A_{00}(e)=A_{01}(e) A_{10}(e)$.

Proof. Since $B$ is an ideal either $B=A$ or $B=0$. If $B=0$ then $A_{10}=A_{01}=0$. Hence $A_{00} A_{00} \subseteq A_{00}$. Thus $A=A_{11}(e) \oplus A_{00}(e)$ and $A_{11}(e), A_{00}(e)$ are ideals of $A$. Since $e \notin A_{00}(e), A_{00}(e) \neq A$. Therefore $A_{00}(e)=0$ and $A=A_{11}(e)$. But this contradicts the assumption that $e \neq 1$. Therefore $B=A, A_{11}(e)=A_{10}(e) A_{01}(e)$ and $A_{00}(e)$ $=A_{01}(e) A_{10}(e)$.

COROllary 2. If $e$ is an idempotent of a simple associo-symmetric algebra $A$ then $A_{00}(e)^{2} \subseteq A_{00}(e)$.

Proof. Let $x, y \in A_{00}(e)$. Then by Corollary $1, x=x_{01} x_{10}$ and $x y=\left(x_{01} x_{10}\right) y$ $=g\left(x_{01}, x_{10}, y\right) x_{01}\left(x_{10} y\right)$. The right-hand side is clearly in $A_{00}(e)$. Therefore the result follows.

LEMMA 3.3. If $e$ is an idempotent of an associo-symmetric algebra $A$ and $A_{i j}$ $=A_{i j}(e)$ then

(a) $\left(A_{10}, A_{01}, A_{11}\right)=0$,

(b) $\left(A_{11}, A_{10}, A_{01}\right)=0$,

(c) $\left(A_{01}, A_{11}, A_{10}\right)=0$.

Proof. The linearization of fourth power-associativity, $\left(x, x, x^{2}\right)=0$, gives

$$
\begin{aligned}
(x, y, z w+w z) & +(z, y, x w+w x)+(w, y, x z+z x)+(y, x, z w+w z) \\
& +(z, x, w y+y w)+(w, x, y z+z y)+(z, w, x y+y x) \\
& +(x, w, y z+z y)+(y, w, x z+z x)+(w, z, x y+y x) \\
& +(x, z, y w+w y)+(y, z, w x+x w)=0
\end{aligned}
$$

Let $x \in A_{10}, y \in A_{01}, z \in A_{11}$, and $w=e$. Then $z w+w z=2 z, x w+w x=x$, and $w y+y w=y$. Also, by Theorem 2.1, xz=zy=0. Therefore for these specializations (7) reduces to

$$
\begin{aligned}
2(x, y, z) & +(z, y, x)+(e, y, z x)+2(y, x, z)+(z, x, y)+(e, x, y z)+(z, e, x y) \\
& +(z, e, y x)+(x, e, y z)+(y, e, z x)+(e, z, x y)+(e, z, y x) \\
& +(x, z, y)+(y, z, x)=0 .
\end{aligned}
$$

Now $(z, y, x)=0$ since $A_{11} A_{0 i}=0,(y, x, z)=0=(x, z, y)$ since $A_{i 0} A_{1 j}=0$. Also $e y=e[y(z x)]=0, x e=e(y z)=0$. Therefore $(e, y z, x)=(x, e, y z)=0$. Also $e x=x$ and $e[x(y z)]=x(y z)$ since $x(y z) \in A_{11}$. Therefore $(e, x, y z)=0$. Similarly $(z, e, x y)$ $=(z, e, y x)=(y, e, z x)=(e, z, x y)=(e, z, y x)=0$. Therefore (8) reduces to

$$
2(x, y, z)+(z, x, y)+(y, z, x)=0 \text {. }
$$


But third power-associativity gives

$$
(x, y, z)+(z, x, y)+(y, z, x)=0 .
$$

Therefore $(x, y, z)=0$ proving that $\left(A_{10}, A_{01}, A_{11}\right)=0$. In $(10)$ again $(z, x, y)+$ $(y, z, x)=0$. But $(z, x, y) \in A_{11}$ and $(y, z, x) \in A_{00}$. Therefore $(z, x, y)=(y, z, x)$ $=0$. Hence $\left(A_{11}, A_{10}, A_{01}\right)=\left(A_{01}, A_{11}, A_{10}\right)=0$.

Lemma 3.4. Under the same hypothesis as the previous lemma, $\left(A_{10}, A_{00}, A_{01}\right)$ $=\left(A_{11}, A_{10}, A_{00}\right)=0$.

Proof. Let $x \in A_{10}, y \in A_{00}$, and $z \in A_{01}$. Then third power-associativity reduces to $(x, y, z)+(y, z, x)+(z, x, y)=0$. But $(x, y, z) \in A_{11}, \quad(y, z, x)+(z, x, y) \in A_{00}$. Therefore $(x, y, z)=0$. Similarly if $x \in A_{11}, y \in A_{10}$, and $z \in A_{00}$ we immediately obtain $(x, y, z)=0$.

THEOREM 3.2. Let $e \neq 1$ be an idempotent of a simple associo-symmetric algebra $A$ over a field of characteristic $\neq 2$. Then $A_{i i}(e)$ is associative for $i=0,1$.

Proof. Let $x, y, z \in A_{11}=A_{11}(e)$. By the corollary to Lemma 3.1, $y=y_{10} y_{01}$ for some $y_{i j} \in A_{i j}$. Then $(x y) z=\left[x\left(y_{10} y_{01}\right)\right] z$. By (b) of the previous lemma $x\left(y_{10} y_{01}\right)$ $=\left(x y_{10}\right) y_{01}$. Therefore $(x y) z=\left[\left(x y_{10}\right) y_{01}\right] z$. Since $x y_{10} \in A_{10}$ and by (a), $\left[\left(x y_{10}\right) y_{01}\right] z$ $=\left(x y_{10}\right)\left(y_{01} z\right)$. Therefore $(x y) z=\left(x y_{10}\right)\left(y_{01} z\right)$. By (b) again and since $y_{01} z \in A_{01}$, $\left(x y_{10}\right)\left(y_{01} z\right)=x\left[y_{10}\left(y_{01} z\right)\right]$. Finally, by $(\mathrm{a}), y_{10}\left(y_{01} z\right)=\left(y_{10} y_{01}\right) z$. Therefore $(x y) z$ $=x\left[\left(y_{10} y_{01}\right) z\right]=x(y z)$ and $A_{11}$ is associative. Since $A_{00}(e)=A_{11}(1-e), A_{00}$ is also associative.

4. Decomposition relative to several idempotents. If $A$ is an alternative or Jordan algebra and $e_{1}, e_{2}, \ldots, e_{t}$ are orthogonal idempotents of $A$, then one has a vector space decomposition $A=\sum A_{i j}(i, j=0,1, \ldots, t)$ with $A_{i j}=\left\{x \mid e_{k} x=\delta_{k i} x\right.$ and $\left.x e_{l}=\delta_{j l} x\right\}$ with $\delta$ the Kronecker delta. We show that the same decomposition is obtained for associo-symmetric algebras.

LEMMA 4.1. Let $e, e^{\prime}$ be orthogonal idempotents of an associo-symmetric algebra $A$ with 1. Then $e\left(e^{\prime} x\right)=\left(x e^{\prime}\right) e=0$ and $\left(e, x, e^{\prime}\right)=0$.

Proof. Let $A=A_{11}+A_{10}+A_{01}+A_{00}$ be the decomposition relative to the idempotent $e$. Then since $e$ and $e^{\prime}$ are orthogonal, $e^{\prime} \in A_{00}$. Thus if $x \in A$ then $x=x_{11}$ $+x_{10}+x_{01}+x_{00}$ and $e^{\prime} x \in A_{00}\left(A_{11}+A_{10}+A_{01}+A_{00}\right) \subseteq A_{01}+A_{00}$. (Here we are using the stronger form of (3) in an algebra with 1; namely, $A_{00}^{2} \subseteq A_{00}$.) Therefore $e\left(e^{\prime} x\right)=0$. Similarly $x e^{\prime} \in A_{10}+A_{00}$ and so $\left(x e^{\prime}\right) e=0$. Now from third powerassociativity either $g\left(e, x, e^{\prime}\right)=1$ or $e\left(x e^{\prime}+e^{\prime} x\right)+e^{\prime}(x e+e x)+x\left(e e^{\prime}+e^{\prime} e\right)=0$ which reduces to $e\left(x e^{\prime}\right)+e^{\prime}(x e)=0$. But $e\left(x e^{\prime}\right) \in A_{10}, e^{\prime}(x e) \in A_{01}$. Therefore $e\left(x e^{\prime}\right)=0$ and $(e x) e^{\prime}=g\left(e, x, e^{\prime}\right) e\left(x e^{\prime}\right)=0$ and in this case also $\left(e, x, e^{\prime}\right)=0$.

In routine fashion the previous lemma gives

THEOREM 4.1. Let $e_{1}, e_{2}, \ldots, e_{t}$ be orthogonal idempotents of an associo-symmetric algebra $A$ with 1 . Then $A=\sum A_{i j}(i, j=0,1, \ldots, t)$ is a vector space decomposition of $A$ with $A_{i j}=\left\{x \mid e_{k} x=\delta_{i k} x\right.$ and $\left.x e_{l}=\delta_{j l} x\right\}$. 
Lemma 4.2. $A_{i j} A_{k l}=0$ if $j \neq k(i, j, k, l=0,1,2, \ldots, t)$.

Proof. Either $j \neq 0$ or $k \neq 0$. If $j \neq 0$ then $A_{i j} \subseteq A_{11}\left(e_{j}\right)+A_{01}\left(e_{j}\right)$. But $A_{k l} \subseteq A_{01}\left(e_{j}\right)$ $+A_{00}\left(e_{j}\right)$. Therefore $A_{i j} A_{k l}=0$. Similarly if $k \neq 0$.

We now prove the following fundamental theorem on associo-symmetric algebras.

THEOREM 4.2. Let $A$ be a simple associo-symmetric algebra over a field of characteristic $\neq 2$ and let $1=e_{1}+e_{2}+\cdots+e_{t}$ for pairwise orthogonal idempotents $e_{i}$. Then if $t>2, A$ is associative.

Proof. We shall be considering the Peirce decomposition $A=\sum A_{i j}$ relative to $e_{1}, e_{2}, \ldots, e_{t}$. Let $e=e_{1}+e_{i}$. Then $A_{11}(e)=e A e=A_{11}+A_{1 i}+A_{i 1}+A_{i i}$ is associative by Theorem 3.2. Therefore $\left(A_{1 i}, A_{i 1}, A_{1 i}\right)=0$. But $A_{10}\left(e_{1}\right)=\sum_{j=2}^{t} A_{1 j}$ and $A_{01}\left(e_{1}\right)$ $=\sum_{j=2}^{t} a_{j 1}$. Now let $a, c \in A_{10}\left(e_{1}\right)$ with $b \in A_{01}\left(e_{1}\right)$. Then $a=\sum_{j=2}^{t} a_{1 j}, c=\sum_{j=2}^{t} c_{1 j}$, and $b=\sum_{j=2}^{t} b_{j 1}$. Then $(a b) c=\sum_{j, k, l=2}^{t}\left(a_{1 j} b_{k 1}\right) c_{1 l}$. By the previous remark if $j=k=l$ then $\left(a_{1 j} b_{k 1}\right) c_{1 l}=a_{i j}\left(b_{k 1} c_{1 l}\right)$. If $j \neq k$ then $a_{1 j} b_{k 1}=0$ by Lemma 4.2. Therefore $(a b) c$ $=\sum_{j, l=2 ; j \neq l}^{t}\left(a_{1 j} b_{j 1}\right) c_{1 l}+\sum_{j=2}^{t} a_{1 j}\left(b_{j 1} c_{1 j}\right)$. But $a_{1 j} \in A_{01}\left(e_{j}\right)=A_{10}\left(1-e_{j}\right), b_{j 1} \in A_{10}\left(e_{j}\right)$ $=A_{01}\left(1-e_{j}\right)$, and $c_{1 l} \in A_{00}\left(e_{j}\right)=A_{11}\left(1-e_{j}\right)$. Since, by Lemma 3.3, $\left(A_{10}, A_{01}, A_{11}\right)=0$ we have $\left(a_{1 j} b_{j 1}\right) c_{1 l}=a_{1 j}\left(b_{j 1} c_{1 l}\right)$. Therefore $(a b) c=\sum_{j, l=2}^{t} a_{1 j}\left(b_{j 1} c_{1 l}\right)$. On the other hand $a(b c)=\sum_{j, k, l=2}^{t} a_{1 j}\left(b_{k 1} c_{1 l}\right)$. But $b_{k 1} c_{1 l} \in A_{10}\left(e_{k}\right) A_{00}\left(e_{k}\right) \subseteq A_{10}\left(e_{k}\right)$ and $a_{1 j}$ $\in A_{00}\left(e_{k}\right)$. Therefore $a_{i j}\left(b_{k 1} c_{1 l}\right)=0$ if $j \neq k$ and $a(b c)=\sum_{j, l=2}^{t} a_{1 j}\left(b_{j 1} c_{1 l}\right)$ also. Thus $(a b) c=a(b c)$. A similar argument shows that $\left(A_{01}\left(e_{1}\right), A_{10}\left(e_{1}\right), A_{01}\left(e_{1}\right)\right)=0$. Thus we have

LeMma 4.3. $\left(A_{10}\left(e_{1}\right), A_{01}\left(e_{1}\right), A_{10}\left(e_{1}\right)\right)=\left(A_{01}\left(e_{1}\right), A_{10}\left(e_{1}\right), A_{01}\left(e_{1}\right)\right)=0$.

Lemma 4.3 together with Lemmas 3.3 and 3.4 is sufficient to prove the associativity of $A$ by showing that all associators $\left(A_{i j}\left(e_{1}\right), A_{k l}\left(e_{1}\right), A_{r s}\left(e_{1}\right)\right)=0$. We show this for several cases which indicate the method to be used in general. Clearly $\left(A_{i j}, A_{k l}, A_{r s}\right)=0$ if $j \neq k$ or $l \neq r$. By Lemmas 3.3, 3.4 and Theorem 3.2, $\left(A_{11}, A_{10}, A_{01}\right)$ $=\left(A_{11}, A_{10}, A_{00}\right)=\left(A_{11}, A_{11}, A_{11}\right)=0$. We show that $\left(A_{11}, A_{11}, A_{10}\right)=0$. $\left(A_{i j}\right.$ indicates $A_{i j}\left(e_{1}\right)$.) Let $x, y \in A_{11}, z \in A_{10}$. Then by the corollary to Theorem 3.1 $y=y_{10} y_{01} \in A_{10} A_{01}=A_{11}$. Then $(x y) z=\left[x\left(y_{10} y_{01}\right)\right] z$. Since $\left(A_{11}, A_{10}, A_{01}\right)=0$, $(x y) z=\left[\left(x y_{10}\right) y_{01}\right] z$. But $x y_{10} \in A_{10}, z \in A_{10}$ and, by Lemma 4.3, $\left(A_{10}, A_{01}, A_{10}\right)=0$. Therefore $(x y) z=\left(x y_{10}\right)\left(y_{01} z\right)=x\left[y_{10}\left(y_{01} z\right)\right]$ using $\left(A_{11}, A_{10}, A_{00}\right)=0$. Finally $y_{10}\left(y_{01} z\right)=\left(y_{10} y_{01}\right) z$ by Lemma 4.3. Therefore $(x y) z=x\left[\left(y_{10} y_{01}\right) z\right]=x(y z)$. We have shown that all associators which have an element of $A_{11}$ in the first place are zero.

We now consider associators having an element of $A_{10}$ in the first entry. We know that $\left(A_{10}, A_{00}, A_{01}\right)=\left(A_{10}, A_{01}, A_{11}\right)=\left(A_{10}, A_{01}, A_{10}\right)=0$ by Lemmas 3.3, 3.4, and 4.3. What remains is $\left(A_{10}, A_{00}, A_{00}\right)$. Let $x \in A_{10}, y, z \in A_{00}$. Then since $A_{00}=A_{01} A_{10}, \quad y=y_{01} y_{10}$ and $(x y) z=\left[x\left(y_{01} y_{10}\right)\right] z$. By Lemma 4.3, $x\left(y_{01} y_{10}\right)$ $=\left(x y_{01}\right) y_{10}$. Therefore $(x y) z=\left[\left(x y_{01}\right) y_{10}\right] z$. But $\left(A_{11}, A_{10}, A_{00}\right)=0$. Therefore $(x y) z$ $=\left(x y_{01}\right)\left(y_{10} z\right)$. Finally we get $(x y) z=x\left[y_{01}\left(y_{10} z\right)\right]$ from $\left(A_{10}, A_{01}, A_{10}\right)=0$. But $y_{01} \in A_{10}(1-e), y_{10} \in A_{01}(1-e)$ and $z \in A_{11}(1-e)$. Therefore $y_{01}\left(y_{10} z\right)=\left(y_{01} y_{10}\right) z$ 
and $(x y) z=x\left[\left(y_{01} y_{10}\right) z\right]=x(y z)$, the desired result. The same arguments are used to show that associators with elements of $A_{01}\left(e_{1}\right)$ or $A_{00}\left(e_{1}\right)$ in the first entry are zero. Therefore $A$ is associative.

5. Semisimple algebras. A power-associative algebra $A$ is called semisimple if its nilradical $=$ maximal nil ideal is zero. If $A$ is a finite-dimensional nonnil algebra than a familiar argument (see [5, p. 39]) shows that $A$ has a principal idempotent $e$. Clearly $e$ is a principal idempotent of $A^{+}$. In [3] Kokoris has shown that $A_{1 / 2}(e)$ $+A_{0}(e) \subseteq \operatorname{Rad} A^{+}$(cf. proof of Lemma 1.1 for notation). But $A_{10}(e)+A_{01}(e)$ $=A_{1 / 2}(e)$ and $A_{00}(e)=A_{0}(e)$. Therefore $A_{10}+A_{01}+A_{00} \subseteq \operatorname{Rad} A^{+}$. Let $x \in A_{10}$, $y \in A_{01}$. Then $2 x \cdot y=x y+y x \in \operatorname{Rad} A^{+}$. Since $y x \in A_{01} A_{10} \subseteq A_{00} \subseteq \operatorname{Rad} A^{+}$we conclude that $x y \in \operatorname{Rad} A^{+}$. Therefore $A_{10} A_{01} \subseteq \operatorname{Rad} A^{+}$. Thus the ideal $B$ $=A_{10}(e) A_{01}(e)+A_{10}(e)+A_{01}(e)+A_{01}(e) A_{10}(e)$ is a nil ideal. If we assume that $A$ is semisimple then $B=0$. Therefore $A_{10}(e)=A_{01}(e)=0$ and $A=A_{11}(e)+A_{00}(e)$. Since $A_{10}=0, A_{00}$ is a subalgebra and the sum is a direct sum $A=A_{11} \oplus A_{00}$. Since $e$ is principal $A_{00}$ is nil. Therefore $A_{00}=0$ and $A=A_{11}(e)$. Therefore $e$ is an identity element of $A$. We have proved the first part of the following theorem.

THEOREM 5.1. Let $A$ be a finite-dimensional semisimple associo-symmetric algebra. Then $A$ has an identity and is the direct sum of simple algebras.

To complete the proof assume that $D$ is an ideal of $A$. Since $D$ is not nil it has principal idempotent $e$. Thus, as before, $D_{10}+D_{01}+D_{00} \subseteq \operatorname{Rad} D^{+}$and $D_{10} D_{01}$ $+D_{10}+D_{01}+D_{01} D_{10}$ is a nil ideal of $D$. Note however that $D_{10}=A_{10}, D_{01}=A_{01}$ and $D_{11}=A_{11}$. For $D_{10}=D \cap A_{10} \subseteq A_{10}$. On the other hand if $x \in A_{10}$ then $x=e x \in D$. Thus $A_{10} \subseteq D$ and $A_{10}=D_{10}$. Similarly for the others. Therefore $B=A_{10} A_{01}+A_{10}+A_{01}+A_{01} A_{10}$ is a nil ideal of $A$. Since $A$ is semisimple $B=0$. Therefore $A=A_{11} \oplus A_{00}=D_{11} \oplus A_{00}$. Since any ideal of $A_{i i}$ is automatically an ideal of $A, A_{i i}(i=1,2)$ is semisimple. Therefore $A$ is a direct sum of semisimple algebras of smaller dimension and an easy induction completes the theorem.

6. We close with a short discussion of the degree one case. If $A$ is a finitedimensional associo-symmetric algebra whose only idempotent is the identity 1 over an algebraically closed field $F$, then an argument of Albert's [2, p. 526] shows that every element $a \in A$ is of the form $a=\alpha 1+n$ with $\alpha \in F$ and $n$ a nilpotent element.

THEOREM 6.1. A finite-dimensional, simple degree one algebra over a field of characteristic $\neq 2$ is a field.

Proof. Assume that $A$ is simple, degree one over $F$. We may assume without loss of generality that $F$ is algebraically closed. Then every $a$ in $A$ is of the form $\alpha 1+n$ and since $A$ is power-associative, if $\alpha \neq 0$ then $a$ has an inverse in $A$. Let $N=\{n \in A \mid n$ nilpotent $\}$. We show that $N$ is a subalgebra, hence an ideal of $A$. By Albert [2] and Oemhke [4], $N$ is a subspace of $A$. Let $x, y \in N$ with $y^{n}=0, y^{n-1} \neq 0$. If $x y$ is not nil- 
potent then $(x y)^{-1}$ exists in $A$. Then $y^{n-1}=\left[(x y)^{-1}(x y)\right] y^{n-1}=g\left((x y)^{-1}, x y, y^{n-1}\right)$ $\times(x y)^{-1}\left[(x y) y^{n-1}\right]=g\left((x y)^{-1}, x y, y^{n-1}\right) g\left(x, y, y^{n-1}\right)(x y)^{-1}\left[x y^{n}\right]=0$. Therefore $y^{n-1}$ $=0$, a contradiction. Hence $x y \in N$ and $N$ is an ideal of $A$. Since $A$ is simple $N=0$ and $A=F 1$.

\section{REFERENCES}

1. A. A. Albert, Power-associative rings, Trans. Amer. Math. Soc. 64 (1948), 552-593. MR $10,349$.

2. - A theory of power-associative commutative algebras, Trans. Amer. Math. Soc. 69 (1950), 503-527. MR 12, 475.

3. L. A. Kokoris, New results on power-associative algebras, Trans. Amer. Math. Soc. 77 (1954), 363-373. MR 16, 442.

4. F. Kosier, On a class of nonflexible algebras, Trans. Amer. Math. Soc. 102 (1962), 299-318. MR 24 \#A3187.

5. R. H. Oehmke, Commutative power-associative algebras of degree one, J. Algebra 14 (1970), 326-332.

6. R. D. Schafer, An introduction to nonassociative algebras, Pure and Appl. Math., vol. 22, Academic Press, New York, 1966. MR 35 \#1643.

Department of Mathematics, Temple University, Philadelphia, Pennsylvania 19122 